\title{
FORO SOCIAL MUNDIAL: DISCURSOS Y GESTOS DE DIVERSIDAD
}

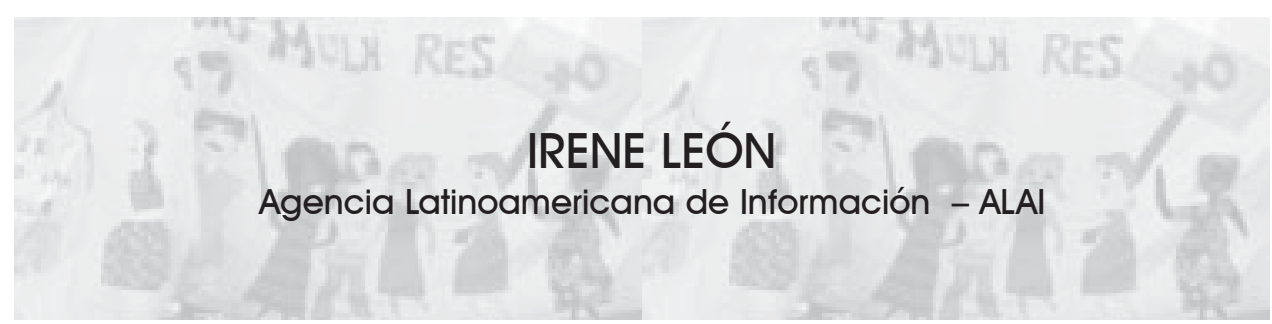

El Foro Social Mundial tomó cuerpo y espacio, justo en un momento en el cual la globalización neoliberal intentaba legitimarse como el destino irreversible y natural de la humanidad, a la vez que sus instituciones presumían de ser los únicos mecanismos capaces de gestionar los intercambios mundiales, las relaciones económicas y también las sociales, culturales y políticas resultantes de sus dinámicas.

A inicios del siglo XXI, fecha de nacimiento del Foro Social Mundial, globalización y neoliberalismo se habían acuñado no sólo como conceptos interreferidos e indisociables, sino como la matriz irrebatible para cualquier iniciativa de intercambio o articulación internacional, colocando al mercado total como hilo conductor, fin en sí y utopía absoluta. $Y$, aunque en todas partes cientos de movimientos y organizaciones sustentaban lo contrario, cundía la versión de que fuera del juego hegemónico de la globalización neoliberal nada era posible ni pensable. Por eso, la simiente de la propuesta de que "Otro Mundo es Posible" fecundó un proceso de interrelación policroma, de apertura de vías para el pensamiento propio y colocó sobre el tapete una multiplicidad de opciones de presente y porvenir.

Según João Pedro Stedile, líder del Movimiento Sin Tierra de Brasil y miembro del Comité Organizador del Foro, "el FSM es un puerto. No es una articulación, su función no es organizar, ni lanzar documentos ni líneas políicas. Pero ha sido un espacio importante para debatir ideas y eso ha sido fundamental en la coyuntura internacional de hegemonía del neoliberalismo y de la ampliación del monopolio de las comunicaciones. Gracias al FSM, los temas sociales, las causas del pueblo volvieron a tener espacio, y fue posible decir que no es verdad que el neoliberalismo es el fin de la historia. El FSM rompió con la hegemonía del 'pensamiento único' que existía en los medios de comunicación, en las universidades, en los medios políticos. Así, la mayor contribución del FSM, ha sido estimular el debate".'

Pero, además, ese laboratorio de ideas que se inauguró desde la primera edición del Foro - Porto Alegre, Brasil, 1991 -, pronto generó un proceso descentralizado y heterogéneo, en el que convergen múltiples actores, acciones y luchas, enfoques temáticos y políticos, convocados por la aspiración común de pensar un mundo incluyente, diverso, pacífico, igualitario y pluralista.

Gracias al FSM es ahora cada vez más visible que las cifras hablan pero no lo dicen todo, pues los pocos 3 mil empresarios y otros hombres de poder que se reúnen en Davos 
(Suiza), o en cualquier otro lugar exclusivo del planeta, pesan autoritariamente más en las definiciones mundiales, pero cualitativamente menos en contenidos y propuestas, que las decenas de miles de personas que se reúnen en Porto Alegre, para pensar en alternativas ante el mundo desigual que los primeros imponen.

"Cinco países toman las decisiones en el Fondo Monetario Internacional. En el Banco Mundial mandan siete. En la Organización Mundial de Comercio todos los países tienen derecho de voto, pero jamás se vota", ${ }^{2}$ señala Eduardo Galeano. Y, los tres mil hombres de poder reunidos, definen la suerte de los 6.314.000.000 de personas que pueblan el planeta, detienen en sus manos la casi totalidad de los recursos financieros, naturales y hasta humanos, e imponen por cualquier vía, incluso la militar, sus diseños y decisiones.

El peso de esos pocos hombres, étnicamente definidos como blancos y socioeconómicamente identificados como ricos, heterosexuales, ganadores y mayoritariamente del Norte geopolítico, es hegemónicamente mayor y cualitativamente menor en términos de iniciativas futuristas que aquel de los más de 3.200.000.000 mujeres que, desde su despoder, abogan desde hace siglos por ganar igualdad a parte entera y crear un mundo sencillamente humano, al igual que lo hacen los pueblos indígenas, afrodescendientes, personas del campo y otras que, suma hecha, son la mayoría de la humanidad, pero que sociológicamente son aún conceptualizadas como minorías.

La omnipotencia de esos cuantos hombres reposa y radica en una acumulación de poder colectada en siglos de construcción minuciosa de un mundo excluyente, diseñado en función de los intereses de esos pocos y legitimado ahora a través de la razón unilineal que impone el pensamiento tecnocrático, que se antepone de fuerza a la policroma expresión de la mayoría.

De allí que el surgimiento del FSM, como espacio de pensamiento propio, además de visibilizar propuestas y alternativas a las aberraciones del modelo, ha permitido la apertura de un paraguas que abriga desde diversas fuerzas sociales de resistencia, de tendencias múltiples, hasta distintas corrientes que, atraídas por la potente fuerza de tracción del llamado pragmatismo del discurso neoliberal, continúan, a nombre del realismo y la eficiencia, suscribiendo a la idea de que los cambios solo podrán ser cosméticos. La gestión de esta convivencia es probablemente uno de los mayores retos que el Foro Social Mundial deberá encarar hacia delante.

\section{Las transversalidades del Foro ${ }^{3}$}

En el corto lapso de su existencia, el carácter abierto y participativo de la convocatoria al proceso del Foro Social Mundial, ha permitido no sólo la interacción entre movimientos e iniciativas de distinto corte, sino también el surgimiento de nuevas alianzas, propuestas y convocatorias, que se expresan en múltiples escenarios y problemáticas, con distintas formas y formatos, cuyas interacciones y acciones sientan el germen de un nuevo movimiento mundial de alternativas a la globalización neoliberal.

Para el movimiento feminista su participación en este espacio reviste múltiples retos y oportunidades. Pues, coherente con los nuevos parámetros que el mismo ha sentado, al reavivar la idea de que "Otro Mundo es posible", el Foro Social Mundial ha adoptado los ejes transversales de género y diversidad en su proceso, abriendo así todo un universo para el desarrollo de estrategias, propuestas, análisis políticos y prácticas de largo alcance.

\footnotetext{
2 Eduardo GALEANO, 2003.

${ }^{3}$ Irene LEÓN, 2003a, cuyos contenidos son una primera versión de esta parte del texto.
} 
Las implicaciones de este compromiso revisten en sí mismas una revolución, pues están relacionadas con una refundamentación de todas las perspectivas sociopolíticas, culturales, económicas, para visualizarlas desde un enfoque inclusivo, contrario al paradigma dominante, que ha tendido a ubicar en centro de prácticas y teorías el referente masculino y blanco, universalizándolo.

Al colocar estos ejes transversales, el Foro Social Mundial plantea el doble reto de, por un lado, romper con las visiones y prácticas andro y etnocentristas, para transitar hacia éticas inclusivas en todos los sentidos. Y por otro, llamar a los movimientos dichos específicos a ampliar su campo de acción y propuesta, para incluir el conjunto de problemáticas sociales en sus enfoques. Asunto que, tratándose del principal espacio de articulación de los movimientos sociales y de desarrollo de alternativas a la globalización neoliberal, se transcribe en un impulso para que el conjunto de movimientos sociales evolucionen hacia el desarrollo de estas visiones en el tratamiento de sus problemáticas particulares y de todos los temas inherentes al desarrollo de alternativas a la globalización neoliberal.

El concepto género, acuñado por las feministas el pasado siglo, está relacionado con la puesta en evidencia de relaciones de poder y desigualdad estructural entre los sexos, cuyas manifestaciones alcanzan todas las esferas de la vida social y privada, a tal punto que su erradicación es parte de los compromisos éticos impostergables de las sociedades y, más aún, de los movimientos comprometidos con el desarrollo de alternativas.

Por su parte, la propuesta de diversidad y pluralidad ha sido planteada por múltiples sectores involucrados en la lucha por la erradicación de todas las formas de discriminación, entre ellos el movimiento indígena, el de afrodescendientes, el LGBT, como una alternativa y ética para un convivir humano de paz.

Pero, hasta aquí, por diversas circunstancias inherentes a las realidades discriminatorias, el enfoque de género ha sido percibido como algo de mujeres y el de diversidad como un asunto de los grupos calificados de minoritarios $y$, aunque los movimientos dedicados a estas causas han producido análisis y propuestas de orden integral, pocas veces estos asuntos han llegado a ser parte nodal de los procesos amplios.

Mientras tanto, las organizaciones de discriminados/as, en la mayoría de los casos, han escogido sus propios caminos y estrategias de lucha, muchas veces en condiciones de aislamiento con relación a otros movimientos y actores. Indígenas, afrodescendientes, mujeres, personas discriminadas por su orientación sexual, y otras, han construido sus movimientos haciendo de cada causa singular su universo de acción, en casos, con poca interacción entre ellos mismos. A la vez, quienes reivindican las causas dichas generales han hecho poco caso a estos procesos.

Por eso, la aplicación de estos ejes transversales en un espacio de la magnitud del Foro Social Mundial, abre un terreno no sólo para el diálogo y la interacción sino para la construcción de un nuevo colectivo social, que de hacer concretos estos propósitos, conducirá al desarrollo de alternativas reales al mundo excluyente y discriminatorio actual. Pues la transversalidad implica desde la participación abierta y plural en los procesos y la toma de decisiones, hasta la puesta en marcha de visiones, análisis y diseños políticos que incluya las cosmovisiones y perspectivas de los/as discriminados/as.

El significado de este proceso en un contexto de incremento de la exclusión social, que resulta del afianzamiento de la globalización neoliberal, se presenta como uno de coherencia y corresponsabilidad para quienes reivindican el cambio social, pues, a estas alturas, el entendimiento de las causas y efectos de las múltiples formas de discriminación, es ineludible incluso para la comprensión de la geopolíica global, la macroeconomía, la rearticulación de lo social y los cambios culturales. 
¿Cómo enfocar, por ejemplo, problemáticas tales como las migraciones o la pobreza sin poner en perspectiva su configuración étnica y de género? ¿Cómo plantear la vigencia de los derechos humanos y de la ciudadanía plena si en las propias dinámicas sociales subsisten rezagos de racismo, homofobia o sexismo? ¿Cómo plantear un mundo diferente si este no se visualiza como inclusivo?

Por eso el reto planteado al conjunto por el Foro Social Mundial es uno de avances colectivos, que permitirá el surgimiento de nuevas dinámicas sociales y de nuevos planteamientos políticos, que coloquen como sujeto de los cambios y de construcción de la igualdad a todas y todos, y que al hacerlo genere discursos y prácticas incluyentes, al fin portadoras de valores forjadores de humanidad.

\section{Balances de las mujeres ${ }^{4}$}

El movimiento feminista viene aportando sus contribuciones desde los inicios de este proceso, con la ventaja comparativa de su historial de movimiento descentralizado y horizontal y de su bagaje de propuestas bastante sincrónicas a las del Foro. Importantes redes y articulaciones de mujeres, como la Marcha Mundial de Mujeres, la Red de Mujeres Transformando la Economía, la Articulación de Mujeres de la CLOC/Vía Campesina, el Dialogo Sur/Sur LGBT, el Área Mujeres de ALAl, y otras, han abierto en los tres Foros mundiales - Porto Alegre 2001, 2002 y 2003 - espacios para formular estrategias concretas, orientadas a afianzar el enfoque de género y diversidad en el proceso. Así mismo, han procurado sobrepasar las líneas trazadas por las crudezas de la discriminación, donde lo específico marcaba territorios y campos de acción delimitados, para posisionar sus enfoques en los llamados temas generales, es decir los de diseño del futuro colectivo.

En el trasfondo de este ejercicio está, sin duda, la constatación de que los grupos discriminados tienen un acceso diferenciado al llamado discurso general, a la participación, a la toma de decisiones y a la visibilidad pública. Así también, dadas las especificidades inherentes a dichas discriminaciones, sus procesos organizativos han sido principalmente volcados hacia sí, por lo que la participación en un espacio abierto no deja de revestir múltiples retos, algunos de ellos nuevos de cara a una iniciativa como la del Foro, que trata de ser también una innovadora construcción colectiva, donde confluyen tanto la voluntad política para propiciar la igualdad como expresiones, acaso inevitables, de una cultura de la desigualdad aún latente.

Así, si bien un paso decisivo ha sido explicitar género y diversidad como ejes transversales del Foro, aún siguen pendientes esfuerzos para que este propósito se haga realidad en las prácticas, metodologías y discursos. Según la brasileña Miriam Nobre, de la Marcha Mundial de Mujeres, "entre las 1700 actividades que cobijó la tercera edición del Foro en el 2003 - paneles, conferencias, seminarios, talleres, mesas de controversia, testimonios -, el segundo tema más tratado fue justamente género, luego de desarrollo sustentable".

Lo que habla por sí mismo del interés que el asunto reviste y de la presencia de actoras que lo impulsan.

Fue notable, señala Nobre, la presencia feminista y la lectura feminista en todos los

\footnotetext{
${ }^{4}$ Irene LEÓN, 2003b. Es una primera versión de esta parte del texto.

${ }^{5}$ Miriam NOBRE, 2003. Presentación en el Seminario Género, Diversidad y Estrategias Frente a la Globalización, organizado por: Articulación de Mujeres CLOC/Vía Campesina; Agencia Latinoamericana de Información ALAI-Mujeres; Diálogo Sur/Sur GLBT; Marcha Mundial de Mujeres y la Red Latinoamericana de Mujeres Transformando la Economía - REMTE.
} 
temas y actividades, no obstante, ésta aparece aún fragmentada, con poca interacción con las otras posturas, a veces incluso como voces paralelas, que aluden apenas a un sector y no al conjunto.

Para la ecuatoriana Magdalena León T., de la Red de Mujeres Transformando la Economía, "esto tiene que ver, en parte, con la relativamente reciente incursión de este movimiento en la toma de posturas frente a la globalización, la economía, la paz mundial u otros temas de humanidad, pues habida cuenta de la magnitud de la desigualdad que afecta a las mujeres, parte substancial de las acciones de su movimiento han sido principalmente autoreferidas. Pero también, esto está relacionado con la cultura y posturas de los otros movimientos, especialmente de aquellos que tienden a mirar los cambios exclusivamente como algo que ocurre a nivel macro, que depende de instancias de poder frente a las cuales se puede presionar pero no decidir y colocan como secundarios los cambios cotidianos que pueden estar en el campo de decisiones posibles, si los movimientos e individuos que los forman se comprometen con ese cambio. En este sentido, nada impide al conjunto de actores del Foro asumir como propio el asunto de la igualdad entre los géneros y la diversidad, sin esperar a transformaciones estructurales". ${ }^{\circ}$

En cuanto a la aplicación del eje transversal de diversidad, Sally Burch, de ALAl, señala que uno de los aspectos más trascendentes del Foro, como proceso y evento, es el acercamiento entre movimientos, la procura de la unidad en la diversidad, que supone la interacción entre variados enfoques, estilos y contenidos. De ahí resulta la expectativa de un nuevo liderazgo integrador y no por segmentación temática. "La diversidad no puede ser vista como una yuxtaposición de actores y temas sino como diálogo, debate y apropiación de una agenda común por parte de todos y todas. Esto tiene un correlato con la comunicación, porque para ello se requiere democratizar las voces en sentido contrario al impuesto por la corporativización de fuentes y medios, que predomina hoy bajo la lógica de control de mercado."7

Victoria Tauli Corpuz, de Iniciativa Indígena por la Paz, agrega por su parte "que ha llegado el momento de que los discursos políticos reflejen la diversidad cultural, pues ésta es parte constitutiva de todas las sociedades y por tanto tiene que ver con el conjunto de temas y posturas, no tan sólo con las manifestaciones artísticas o los asuntos lingüísticos". ${ }^{8}$

Mientras que Blanca Chancosa, indígena quichua de la CONAIE y del Foro Social Américas, añade que es evidente aún una débil participación de mujeres indígenas en el Foro y que esta tiene que ver también con situaciones locales y a veces vernaculares que limitan su presencia y liderazgo políico, lo que se refleja en este espacio. "El acceso de las mujeres a la decisión y liderazgo es aún percibida como de segundo plano, en reemplazo a la figura masculina."

Así, los retos son múltiples y de alcances diferentes, entre ellos, el señalado por la sudafricana Phumi Mtetwa, del Dialogo Sur/Sur LGBT, "es clave que los movimientos de mujeres y por la diversidad, debatan e interactúen aún más con los otros, sin eludir los temas polémicos ni soslayar las diferencias". ${ }^{10}$

De allí que, luego de constatar la visibilidad y presencia, en términos cuantitativos y cualitativos, de los asuntos de género y diversidad, es un reto actual hacer que las visiones e ideas relacionadas atraviesen de verdad al conjunto de actoras y actores del Foro; que

\footnotetext{
${ }^{6}$ Magdalena LEÓN T., 2003.

${ }^{7}$ Sally BURCH, 2003.

${ }^{8}$ Victoria TALULI CORPUZ, 2003.

9 Blanca CHANCOSA, 2003.

${ }^{10}$ Phumi MTETWA, 2003.
} 
esto sea parte de los procesos regionales de manera amplia y no más un debate considerado sólo de mujeres y de los otros discriminados. Asimismo, desde luego, existe un reto político mayor que es el de articular las diversas corrientes del movimiento de mujeres e interactuar, a la vez, con otros movimientos, sin perder la autenticidad.

\section{Conclusiones}

El surgimiento e impacto del Foro Social Mundial es la muestra más elocuente de que "si bien el siglo nació en medio de una evidente agudización de las crisis que genera el neoliberalismo, es innegable que lo hizo también en medio de una procura de sinceramiento de múltiples fuerzas sociales, que están colocando firme y fuerte la necesidad de cambiar el paradigma del capital por uno de humanidad"."

Entre los actores de este proceso figura el movimiento feminista, reconocido por un trayecto de cuestionamientos a las relaciones de poder, los paradigmas dominantes y las prácticas organizativas verticales; que tiene como activo, además, uno de los mayores logros humanos de la historia reciente: la obtención de derechos universales para las mujeres, cuya concreción, sin embargo, está en riesgo por el carácter excluyente de la globalización neoliberal.

"La visible omisión de la perspectiva de género en el delineamiento de la economía neoliberal mundial da cuenta de una regresión potencial. Siendo que su propio diseño, como lo evidencian los acuerdos como los de libre comercio, obvia la situación de las mujeres. Proponer la inclusión de un agregado de género o de una cláusula social no basta. Para que estos tengan enfoque de género tendrían que ser rediseñados, colocando lo humano y en ello la situación de las mujeres al centro de sus preocupaciones. No siendo así, participar de las resistencias contra los acuerdos de la Organización Mundial de Comercio o los Acuerdos de Libre Comercio de las Américas es un asunto específico de mujeres", como lo son todos los relacionados con la economía y, más aún, el conjunto de aspectos inherentes a la globalización.

Pues, si hasta hace poco los posicionamientos sobre la globalización aparecían como alejados de las preocupaciones de las mujeres, ya no lo son más. El avance acelerado de ese proceso, abarca todos los aspectos de la vida, los cotidianos, los macrosociales, los económicos, los culturales, todos en absoluto, y todos ellos tienen que ver con las mujeres. Entonces, desarrollar enfoques feministas para una globalización solidaria y diferente no es un asunto retórico, es parte de la propia evolución de esa propuesta y su ubicación en el contexto.

Así, para todas las mujeres y para el movimiento feminista en particular, la participación directa en los espacios colectivos de creación de modelos que permitan la puesta en práctica de sus derechos, lo que está relacionado con cambios societales globales, es un punto de agenda ineludible, que implica la transición hacia una nueva manera de pensar y hacer las cosas, ampliando lo planteado en los decenios pasados hacia el universo de los llamados temas generales. Pues, todo balance hecho, es justamente allí donde se ubican los cuellos de botella del ejercicio de derechos y la ciudadanía de las mujeres. Ello con mayor razón si los mecanismos excluyentes de la globalización neoliberal se potencian justamente en la combinación de las múltiples formas de discriminación preexistentes, entre las cuales la desigualdad entre los géneros es una de las de mayor

\footnotetext{
${ }^{11}$ Irene LEÓN, 2002a.
} 
masividad.

Por tanto, la inclusión de las propuestas feministas en escenarios como el del Foro Social Mundial, donde se gestan ideas para el diseño de una globalización diferente, la participación del movimiento en la configuración de las alianzas, en la creación de los discursos críticos y de las propuestas nuevas, es un esfuerzo necesario para que la visualización de un mundo diferente sea incluyente y tenga enfoque de género.

A estas alturas, en un momento marcado por el empinamiento de lo económico como ideología, donde la cultura, la política, el cotidiano, lo individual, lo colectivo, todo se percibe desde la óptica de una dinámica cada vez más focalizada en los éxitos del capital; en un momento en que los márgenes de exclusión que genera el neoliberalismo son de lo más elocuentes, las soluciones no pueden apenas ser cosméticas sino que, como lo plantea el Foro Social Mundial, tienen que encarar alternativas reales y en ello el movimiento feminista tiene mucho que aportar.

\section{Referencias bibliográficas}

ALAI. "La comunicación en el Foro Social Mundial". Documento de la web 2003: $<$ www.alainet.org $>$.

BURCH, Sally. Presentación en el Seminario Género, Diversidad y Estrategias Frente a la Globalización. Foro Social Mundial, Porto Alegre, Brasil, enero 2003.

CHANCOSA, Blanca. Presentación en el Seminario Género, Diversidad y Estrategias Frente a la Globalización. Foro Social Mundial, Porto Alegre, Brasil, enero 2003.

GALEANO, Eduardo. "Los valores sin precio". Documento de la web 2003: <www.alainet.org/ active/show_text.php3?key $=3099>$. Palabras pronunciadas por el escritor en el tercer Foro Social Mundial, Porto Alegre, Brasil, 26 de enero de 2003.

LEÓN, Irene. Pluralizar el mundo, diversificar las voces. Ecuador: Ed. ALAl, 2001. . "Apuntes para una crítica feminista del neoliberalismo". Documento de la web 2002a: $<$ www.alainet.org $>$.

"Un mundo diverso es posible". Documento de la web 2002b: <www.alainet.org >. (ed.). Challenges for Feminism in a Globalized World. Ecuador: ALAl and NOBIV, 2002c. . "Las transversalidades del Foro Social Mundial". Documento de la web 2003a: <http:/ /www.alainet.org/active/show_text.php3?key=3016>.

. "Miradas de género y diversidad en el FSM". ALAI/Minga Informativa. Documento de la web 2003b: <www.movimientos.org/fsm>.

LEÓN, Irene; LEÓN, Magdalena. Mujeres contra el ALCA: razones y alternativas. Ecuador: Ed. ALAI, 2002.

LEÓN, Irene; MTETWA, Phumi. Globalización: alternativas GLBT. Ecuador: Dialogo Sur/Sur GLBT, 2003.

LEÓN T., Magdalena. Presentación en el Seminario Género, Diversidad y Estrategias Frente a la Globalización. Foro Social Mundial, Porto Alegre, Brasil, enero 2003.

MAGRISSO, Larissa. "Joao Pedro Stedile nos marcos da realização do III FSM". Documento de la web 2003: <http://www.alainet.org/active/show_text.php3?key=3045>. Entrevista realizada en enero 2003, Portal Terra, Porto Alegre-RS.

MOVIMIENTOS SOCIALES. "Llamamiento de los Movimientos Sociales". Documento de la web 2003: <www.movimientos.org/fsm2003/>.

MTETWA, Phumi. Presentación en el Seminario Género, Diversidad y Estrategias Frente a la Globalización. Foro Social Mundial, Porto Alegre, Brasil, enero 2003.

NOBRE, Miriam. Presentación en el Seminario Género, Diversidad y Estrategias Frente a la Globalización. Foro Social Mundial, Porto Alegre, Brasil, enero 2003. 
TALULI CORPUZ, Victoria. Presentación en el Seminario Género, Diversidad y Estrategias Frente a la Globalización. Foro Social Mundial, Porto Alegre, Brasil, enero 2003.

World Social Forum: speeches and gestures of diversity

Abstract: The text presents the World Social Forum as a laboratory of ideas, that has generated a decentralized and heterogenious process, with many actors/ actresses, political and thematic focus. The inclusion of feminist proposals in this scenario, where ideas for the reshaping of a different globalization are generated, the participation of the feminist movement in the building of alliances, in the creation of critic opinions and of new proposals, is a necessary effort so that the projection of a different world can be includent and have gender perspective.

Key words: feminist proposals, alliances, political actresses. 